\title{
Expériences migratoires de jeunes Ouest-Africains en France
}

\author{
Alani Souleymane, University of Ibadan, Nigeria \\ Nathalie Agbessi, University of Ibadan, Nigeria
}

L'immigration étrangère en Europe prend de l'ampleur, alors que les tragédies liées à la traversée clandestine de la Méditerranée et les camps d'esclaves en Libye se multiplient. Les médias présentent « une victimisation des migrants renvoyant à une vision misérabiliste de la migration » (Schmitz 5), à l'image du reportage de Nima Elbagir sur la vente de migrants subsahariens en Lybie publié par CNN en 2018. Des jeunes, hommes et femmes, sont réduits en esclaves dans leur tentative désespérée de se rendre en Europe. Cette tragédie avive le débat entre la légalité et l'illégalité de la migration, mais nécessite des recherches sur les expériences des migrants subsahariens qui ont réussi la traversée de la Méditerranée, concernant, parmi d'autres, certains jeunes francophones de l'Afrique de l'Ouest qui ont choisi la France comme destination.

L'examen des phénomènes migratoires de ces jeunes ouest-africains vise la documentation de leurs motivations, du choix de la France comme destination, des difficultés rencontrées pour effectuer le voyage, des problèmes d'intégration et des fonds transférés aux proches. La collecte de données est faite à l'aide de questionnaires et d'entretiens fournis par quelques jeunes migrants ouest-africains résidant en France depuis au moins six mois. Les réponses sont analysées à la lumière des théories migratoires telles que le modèle d'Everett Lee, la théorie néoclassique de la Nouvelle Economie de la migration rattachée à l'économiste Oded Stark, les approches par réseaux traitant du rapport les lieux de départ et d'arriver, et des hypothèses sur les transferts de fonds migratoires.

\section{Immigration et trajets migratoires}

Cette étude se base sur les caractéristiques de la migration définies par Paul Boyle (34) et implique notamment le mouvement d'une personne, la durée de résidence et la traversée d'une frontière, pour traiter la migration internationale. En effet, plusieurs facteurs favorisent la migration : les catastrophes naturelles, la surpopulation qui produit les problèmes d'emploi, de logement, d'infrastructures et autres; les conflits armés ; la mauvaise gouvernance et la pauvreté. Parmi les migrants internationaux, qui sont en situation soit régulière soit irrégulière, on dénombre des réfugiés, des personnes déplacées, des migrants économiques et des 
personnes qui se déplacent pour des raisons comme le regroupement familial (voir Glossary on Migration).

Les migrants de contrainte ont un statut de réfugié et la possibilité de demander l'asile dans les pays d'arrivée. Pour les migrants volontaires qui partent en France pour poursuivre leurs études ou rejoindre un parent, il est beaucoup plus facile d'obtenir un permis de séjour tandis que ceux qui n'ont pas cette opportunité sont poussés à la clandestinité.

\section{Panorama de l'immigration ouest-africaine en France}

L’immigration africaine en France débute avec la venue des Algériens vers la fin du dix-neuvième siècle. Les Sub-sahariens arrivent en France après la deuxième guerre mondiale, ce qui « concerne surtout des pays autrefois sous administration française » (Plazaola et Rignols 37). Cependant, depuis quelques années, le nombre de Sub-sahariens en partance pour la France commence à surpasser le taux des Maghrébins. Ces nouveaux migrants sont originaires du Sénégal, du Mali, du Congo, de la Somalie. La plupart sont d'Afrique de l'Ouest (Barou 6).

Pour les migrants francophones ouest-africains, la France demeure la destination de prédilection. Les premières migrations ouest-africaines en France ont débuté au début des années soixante. Elles étaient à la base des migrations de travail temporaires. Cependant, de nombreux tirailleurs sénégalais étaient déjà et sont restés en France après les deux guerres mondiales. Ils joueront un rôle important dans l'arrivée et l'installation des migrants travailleurs ouest-africains auxquels ils fourniront aide, conseils et soutien. Après quelques années de travail, ils repartaient dans leur pays natal et faisaient venir leurs cadets pour prendre leur place en France (Diarra 885).

En 2003, Jean-Pierre Garson et Anaïs Loizillon élaborent un panorama des flux migratoires des années cinquante aux années 2000. À partir des années soixante-dix, on y observe un «flux de regroupement familial »(4) : les immigrés préalablement installés en France commencent par faire venir leur femme et leurs enfants. On observe également des demandeurs d'asile composés d'étudiants, de réfugiés politiques et des aventuriers (5). On constate un rajeunissement de la composition des flux migratoires et une plus forte proportion de femmes. De temporaires, les migrations deviennent, à cette époque, définitives. Les années quatre-vingt-dix enregistrent des migrations nuptiales : arrivés à l'âge de se marier, de nombreux immigrés de la seconde génération, dont la plupart sont des hommes, vont chercher leur conjoint dans leur pays d'origine et le font venir en France. 
Les migrations des années 2000 jusqu'à nos jours sont des migrations urbaines, avec des migrants disposant « d'un taux de scolarisation relativement élevé » (Barou 13). Certains migrants proviennent de classes sociales assez privilégiées. Ils migrent grâce à leur niveau élevé d'éducation. Cependant, un nombre considérable de jeunes moins éduqués et frustrés par la pauvreté tentent l'aventure à leurs risques et périls. Ils constituent la majorité des candidats à l'immigration clandestine qui, s'ils arrivent à destination, deviennent des immigrés sans papiers exploités par des employeurs douteux.

En 2018, 46,1\% des immigrés vivant en France sont d'origine africaine selon les estimations de 1'Insee parues en 2019. Selon Michel Foucher, lors d'un entretien avec Laurent Dumas en 2018, « Au Sénégal, par exemple, 70\% des familles ont un membre qui a migré à l'étranger » (par. 3). De même, les jeunes migrants ont le désir de circuler et d'aller améliorer leur niveau de vie dans des pays occidentaux dont ils parlent la langue, en général.

Une autre caractéristique de cette nouvelle migration est la féminisation du flux migratoire. Davantage de femmes rejoignent leur conjoint à l'étranger et d'autres deviennent de plus en plus autonomes (Vause et Tona 62). Les effets économiques des migrations s'expliquent par les flux monétaires envoyés par un migrant vivant dans un pays d'accueil vers un agent dans son pays d'origine, attestant alors « de son attachement et de la force des liens familiaux transnationaux » (Bréant 31).

\section{Théories migratoires et recueil des avis des migrants}

La théorie migratoire de Lee, émise en 1966, examine les facteurs répulsifs et attractifs entrainant la migration. Selon Lee, avant de prendre la décision de migrer, l'individu considère quatre facteurs essentiels : les facteurs liés aux pays d'origine, les facteurs liés aux pays de destination, les obstacles intermédiaires et d'autres facteurs d'ordre personnel (50). Il regroupe ces facteurs en deux différentes catégories qu'il appelle facteurs attractifs et répulsifs (50). Les facteurs répulsifs font référence aux conditions qui peuvent pousser les individus à quitter leur foyer et sont associés aux pays de départ. Les facteurs attractifs font référence aux pays de destination. Ils attirent les migrants vers une certaine location. Il existe également des facteurs « neutres » laissant la plupart des individus indifférents. Toutefois, la décision de partir ne dépend pas uniquement des différents facteurs (attractifs, répulsifs et nuls) mais aussi de la manière dont les migrants potentiels perçoivent ces facteurs. Leur perception est construite grâce aux réseaux de contacts de l'individu et grâce aux différentes sources d'informations présentes dans le pays d'origine. Bref, toute analyse de phénomène migratoire doit s'intéresser au pays d'origine du migrant, aux différents facteurs qui expliquent sa volonté de partir, aux 
obstacles qui peuvent rendre difficile ou empêcher le déplacement de la personne immigrante et au pays d'accueil (plus particulièrement aux éléments qui le poussent à choisir une destination particulière).

La nouvelle économie de migration (NEM) entrevoit la décision de migrer comme un choix collectif, et non plus individuel (Gubert 98). Une famille ou communauté sponsorise le départ d'un migrant dans l'espoir d'amélioration de son statut socio-économique, ou d'une simple assurance vieillesse des parents, ou qu'il les aide à migrer une fois bien établi à l'étranger (Boyd 642). L'approche « réseaux » du phénomène migratoire, telle qu'appliquée par Niandou Touré (2) stipule que la présence d'un réseau migratoire, c'est-à-dire d'un nombre de migrants servant d'acteurs intermédiaires, dans la zone d'accueil encourage de nouveaux départs (3).

Les transferts monétaires constituent l'un des vecteurs clé de l'impact de l'émigration dans les pays en développement et des liens entre les migrants et leurs zones de départ (Mouhoud 223, 226 ; Vargas-Lundius et al. 14). Parmi les motifs qui animent les migrants à transférer de l'argent à leurs proches restés dans les pays d'origine, l'altruisme et l'investissement sont les raisons majeures des transferts de fonds (Azizi 1218). Dans ce cas, le migrant peut transférer de l'argent en contrepartie de l'investissement de la famille dans son éducation et/ou sa migration (Stark et Bloom 175).

A l'aide de questionnaires et entretiens, la présente étude interroge trente migrants ouest-africains de nationalités togolaise, béninoise, sénégalaise, guinéenne, ivoirienne et tchadienne qui ont pleinement vécu le processus migratoire depuis le départ de l'Afrique de l'Ouest. Ce sont vingt hommes et dix femmes installés en France : une personne pour 1998, 2009, 2013, 2014 ; cinq migrants en 2015, deux en 2016, et surtout la majorité qui est récente, à savoir onze en 2017 et huit en 2018. Cinq migrants sont dans la tranche d'âge des 15-20 ans, dix-huit dans celle de 21-25, cinq entre 25-30 ans et deux entre 31-50. La quasi-totalité des participants sont des jeunes de moins de trente ans (96,7\%). Ils sont arrivés en France par avion (93,3\%), ce qui laisse entrevoir que la plupart sont des migrants légaux. Un migrant est arrivé par la route et un autre migrant a décliné de dévoiler sa voie d'entrée en France. Ces trente migrants ont répondu à des questionnaires structurés et distribués sur les réseaux sociaux Facebook et WhatsApp entre le 21 et le 23 février 2019.

Chaque questionnaire contient trois sections. La première entretient des informations de base sur le sexe, l'âge, la nationalité, le lieu de naissance, l'année d'arrivée en France, le lieu de résidence en France et l'objectif migratoire. La deuxième partie examine la cause du choix de la France comme lieu de destination, les sources de financement, le moyen de 
transport, l'influence des parents et bien d'autres facteurs entrant en jeu dans le processus de la migration. La dernière section recueille les effets économiques de la migration sur les pays de départ, les obstacles rencontrés par les migrants en France, le contact de différentes cultures et, en dernier lieu, la possibilité de retour au pays d'origine.

Six parmi les migrants âgés de vingt à trente ans, se sont portés volontaires, sous condition d'anonymat, pour un entretien de type semi-directif. Ils résident tous en France au minimum depuis six mois au moment de l'entretien. Ils acceptent de parler et de donner des informations sur leurs expériences migratoires. Trois questions, relativement ouvertes, ont été posées pour guider et centrer leurs interventions sur l'objectif de l'étude : «Parlez-nous de votre expérience migratoire et des difficultés que vous avez rencontrées », «Pensez-vous que la France est un Eldorado pour la jeunesse africaine ? » et « Quels conseils avez-vous à donner aux jeunes qui souhaiteraient migrer comme vous ?».

Les questions ont été posées en ligne aux migrants. Ils ont fait part de leurs réponses à partir d'enregistrements audio. La durée de chaque réponse a été de dix minutes. Les données des questionnaires ont été traitées de manière descriptive.

\section{Les éléments déterminant la migration}

L'instabilité politique (20\%), la pauvreté, des services de santé défaillants et le goût de l'aventure (13\% respectivement) dans les pays d'origine constituent des facteurs répulsifs Push poussant les jeunes à émigrer. Selon le classement des universités de 2019 par la compagnie britannique Quacquarelli Symonds, aucune université francophone ouest-africaine ne se trouve au top cinquante des universités africaines, d'où l'exode des migrants vers des universités plus réputées en France pour une formation de pointe (73,3\%), qui pourrait aider à trouver de meilleures offres d'emploi et à avoir un niveau de vie élevé. En outre, les universités francophones offrent des parcours d'études limités : «Par rapport à d'où je viens, le système éducatif (en France) est beaucoup plus élaboré, c'est pour ça généralement qu'on quitte l'Afrique » (Répondant 6).

Les migrants décrient la gestion des bourses d'études dans leurs pays d'origine. Après l'octroi des bourses, les gouvernements manquent à leurs engagements. Manque de ressources financières, incompétence administrative, détournement de fonds publics, instabilité politique, coups d'états, soulèvements populaires, répression de l'opposition, républiques transformées en monarchie (où le siège présidentiel est transformé en trône et le pouvoir passé de père en fils), des présidents « éternels » : tous les problèmes se conjuguent pour priver aux étudiants boursiers de leurs droits. Le Répondant 3 atteste : 
Je connais plusieurs personnes qui ont bénéficié de la bourse togolaise et qui viennent ici et qui souffrent vraiment beaucoup parce que le Togo, c'est un pays qui n'honore pas ces engagements, c'est une vérité que j'aimerais dire aussi ; c'est un pays qui n'honore pas ces engagements, le Togo. Ils offrent des bourses aux étudiants, ils les laissent partir et lorsqu'ils partent maintenant ils sont là et regardent et attendent ; ils ne font pas les virements, ils ne se préoccupent pas de comment un enfant, il va vivre, quand il va arriver.

Pour le Répondant 5, la corruption règne également dans ces états et, souvent, seule une infirme partie de la population profite des richesses ses pays : « Il y a plusieurs opportunités en Afrique également si tout n'est pas bloqué, il y a une minorité qui profite, qui en jouit ».

Les opportunités d'emploi, la possibilité d'effectuer une formation de pointe, le niveau de vie élevé et la stabilité politique constituent des facteurs qui attirent les jeunes migrants vers la France :

J'aimerais bien que les gens [...]; étudiants y viennent pour continuer leurs études parce que la plupart des écoles ont des partenaires dans le monde entier ce qui fait que les écoles ont également des connections avec le monde industriel, ce qui fait que surtout on trouve très rapidement des stages à faire pour pouvoir s'acclimater avec le monde professionnel... (Répondant 2)

La persévérance et le combat ouvrent la porte de l'emploi aux migrants : « Ici, il y a du travail, si tu te bats tu trouves du travail» (Répondant 5). En outre, l'abondance et la variété des emplois sont plus présentes en France :

Je prends le cas de ma filière quelqu'un qui travaille en finance, les opportunités de carrière au Togo se limitent principalement à une carrière en audit ou en comptabilité ou bien en tant que banquier privé principalement alors qu'ici le panel de postes ou d'emplois possible est bien plus élargi parce qu'ils ont un marché financier bien établi donc il y a tous les emplois que propose la finance des marchés qui sont accessibles. (Répondant 1)

Etre en France permettrait également de travailler au sein de grandes compagnies et d'entreprises connues dans le monde : « J'aimerais bien travailler dans l'aéronautique, c'est un domaine que j'adore, notamment dans des grands groupes industriels que sont Safran, AirFrance que l'on connait tous d'ailleurs » (Répondant 2). En France, la rémunération des employés est plus élevée : «Il est clair que les salaires en France sont beaucoup plus intéressants que les salaires au Togo, à niveau d'équivalence quelqu'un qui travaille en France, il recevra plus que celui qui travaille au Togo » (Répondant 1).

Le niveau de vie et le développement de la France encouragent la migration : Il y en a qui viennent et qui se retrouvent à faire des travaux misérables, mais la différence c'est que la somme qu'ils gagnent avec ce travail misérable est plus que la somme qu'ils gagneraient s'ils faisaient le même travail dans leurs pays d'origine parce que le niveau de vie est différent. (Répondant 4)- 
Selon les réponses aux questionnaires, l'attrait de la France se fait également ressentir au niveau du développement des infrastructures : « déjà pour les transports en commun à Lomé, les transports communs ne sont pas du tout développés, il y a des taxi-motos, des taxis mais là, il y a le bus, le tram, le métro, bref le total » (Répondant 4). Le niveau de vie élevé permettrait également de mener une vie assez confortable :

J'ai eu la chance d'aller au cinéma, chose que je ne faisais pas avant. Je suis allé au cinéma plusieurs fois. On a aussi la chance d'avoir tout le temps la connexion pour pouvoir faire nos recherches à nous pour pouvoir évoluer chose qu'on a pas tout le temps en Afrique. (Répondant 2)

Le fait que le niveau de vie soit beaucoup plus intéressant pour le fait qu'ils ont une technique de médecine très avancée et qui nous permet de vivre plus longtemps. (Répondant 1)

Les migrants affirment qu'avec la stabilité politique, les emplois sont à la portée de tous : «En France, le jeune Africain a autant d'opportunités que le jeune Français, que le jeune Européen » (Répondant 5).

Parallèlement à la déclaration de Foucher dans l'entretien accordé par Dumas, qui stipule que « ces jeunes [migrants] sont de plus en plus informés », seuls sept migrants avaient une perception fallacieuse de la France - pays d'Eldorado - avant d'émigrer :

En général, le problème, c'est que ce qu'on nous montre à la télé, c'est qu'on nous montre la France comme un pays idéaliste, le pays «parfait »; quand tu entres, tu sors de la pauvreté, on te met bien. (Répondant 4)

Les jeunes candidats à l'immigration ont tendance à idéaliser la France et les autres pays occidentaux en général, où ils pensent que leurs problèmes seront d'emblée résolus : « Nous jeunes, jeunes Africains, on rêve déjà d'aller dans des pays américains, dans des pays européens pour se créer une vie meilleure et pour s'établir un niveau de vie élevé » (Répondant 3). $90 \%$ des migrants pensent que la France offre de meilleures opportunités aux migrants. Pour les vingt-et-un migrants qui ont décidé d'expliquer leur opinion, la France est belle et stable ; elle offre des emplois, une formation spéciale, des infrastructures, l'égalité et une meilleure rémunération. Pendant l'entretien, cinq répondants ont validé leur opinion de la France : les présomptions telles que le niveau normal de recherche, un climat difficile, un beau pays, de bons profs, la diversité culturelle, la facilité de vie et l'individualisme sont vraies (60\%). Par contre, les idées préconçues telles que la prévalence du racisme et la facilité de la vie sont fausses $(40 \%)$.

Les réseaux migratoires jouent un rôle décisif dans le choix migratoire. La décision de migrer est personnelle chez $80 \%$ des migrants répondants. Quand la décision de migrer est prise, les migrants sont influencés partiellement $(23,3 \%)$ ou totalement $(26,7 \%)$ par leurs parents. 
Une fois la décision de migrer prise et annoncée, le processus migratoire, dans le cadre de la nouvelle économie migratoire, est financé par les parents des migrants $(63,3 \%) ; 26,7 \%$ voyagent à leurs propres frais et $10 \%$ grâce à une bourse. Une fois en France, les migrants $(90 \%)$ comptent s'installer définitivement en France, et certains (53,3\%) espèrent travailler et pouvoir s'occuper de leurs parents.

Vingt migrants sur vingt-neuf, soit $69 \%$, ont des parents ou proches qui sont déjà installés en France. Néanmoins, la présence de famille en France a influencé 57,1\% dans leur choix de ce pays comme destination. $69,2 \%$ des migrants reçoivent l'aide de leurs proches à leur arrivée en France, à savoir l'hébergement, la quête de logement, l'intégration sociale et les renseignements sur les démarches administratives à suivre :

En France, j'avais déjà un ami que je considère déjà comme un frère qui m'a bien accueilli, tout s'est bien passé et il m'a conduit de Paris jusqu'à Reins et enfin je me suis bien installé. (Répondant 2)

La présence de parents en France est alors une sorte de réconfort pour les migrants (80\%). Il existe une certaine solidarité entre les anciens et les nouveaux ressortissants du même pays d'origine à l'étranger :

Quand je suis arrivé, il y a plusieurs Togolais qui m'ont contacté, qui étaient à Reims également et on a même fait une petite association de Togolais à Reims ; ce qui a fait que j'ai eu plus l'occasion de me sentir en famille ; et aussi chez les ressortissants d'Afrique Francophone en général. Et généralement chez les Africains originaires de pays francophones. (Répondant 3)

J'ai beaucoup d'amis ici, pas que les Togolais : des Gabonais, des Sénégalais, des Camerounais ; on se côtoie déjà tous ici et c'est très bien entre Africains de pouvoir s'aider, s'entraider tu vois, et c'est déjà un bon point, un truc que j'aime beaucoup. (Répondant 2)

\section{Expérience des migrants en France}

Les obstacles rencontrés par les migrants dans le processus migratoire sont d'ordre consulaire dans le pays d'origine des migrants ; en France, l'intégration culturelle, sociale, le logement et le climat constituent les obstacles principaux :

Avant de quitter le Togo, j'ai eu plusieurs difficultés concernant le visa ... c'est juste que, administrativement parlant, c'était un peu lent donc, comme obstacles, je peux parler des difficultés consulaires. (Répondant 3)

C'était un calvaire. Après on sait qu'il y a toute la paperasserie et tout, pour avoir le visa et tout, pour moi, ça a été le plus difficile. (Répondant 6)

La majorité des répondants admettent que les difficultés en France sont énormes. Les plus courantes sont le froid, le logement, les aliments, le dépaysement, la dépression, 
l'isolement, la présence d'une nouvelle mentalité et l'intégration à une nouvelle culture, à un nouveau mode de vie :

Metz et c'est une grande ville de France et il faut déjà savoir se repérer s'orienter parce qu'il y a pas mal de trams de bus, il faut savoir faire des correspondances parce qu'il y a plusieurs arrêts pour pouvoir aller d'un point à l'autre, donc c'est pas aussi facile que ça. (Répondant 2)

Certains migrants éprouvent des difficultés à s'adapter à la gastronomie française :

Tu ne vas pas trouver des bananes plantains, de l'attiéké à n'importe quel bout de rue comme à Lomé. (Répondant 4)

La gastronomie également... c'est carrément différent, au début je ne consommais que mes propres aliments. (Répondant 2)-

Les migrants rencontrent des difficultés pour trouver un logement, du fait de leur statut d'étranger :

Il est compliqué de trouver des logements dans les grandes villes et surtout quand tu es étranger et noir parce que la plupart des propriétaires, ils n'aiment pas les étrangers ; ils ont peur que ces personnes-là ne puissent pas payer le loyer ; quand tu es étranger, c'est un peu compliqué. (Répondant 4)

L'adaptation au froid est difficile pour les Africains qui arrivent d'un climat tropical :

Au Togo, on est en moyenne à $37^{\circ}$ alors que, ici, les températures sont beaucoup plus basses, et $37^{\circ}$, c'est vraiment un luxe. En général ici, il fait beaucoup plus froid, on est à $20^{\circ}$ dans les beaux temps un temps en moyenne, donc, il fait beaucoup plus froid, ce qui fait que le style vestimentaire, le mode de vie, tout ça change, on se met dans des pulls, on est obligé de dormir dans de grosses couvertures, on est obligé de porter des bonnets. (Répondant 1)

Avec le climat, suit également un dérèglement de saisons :

Je suis arrivé en octobre ; c'était proche de l'automne et on était déjà en hivers, et c'est un changement radical de climat, et l'Hivers c'est surtout au nord de la France, et c'est vraiment terrible. Il fallait déjà s'adapté à ça déjà ; en hiver il fait nuit très vite et déjà à 17 heures 16 heures il fait nuit et $c$ 'est pas aussi facile que ça de s'adapter à ça. (Répondant 2)

Les réponses des migrants aux questions sur les raisons de leurs transferts de fonds vers leurs proches en Afrique valident l'hypothèse selon laquelle les migrants envoient de l'argent à des fins purement altruistes. Ainsi, 46,7 \% des migrants affirment qu'ils travaillaient. Parmi ceux qui envoient de l'argent à leurs proches, 40\% envoient de l'argent mensuellement, $40 \%$ occasionnellement et $10 \%$ rarement pour payer la scolarité des frères et sœurs, pallier la pauvreté ou encore faire plaisir à leur famille. 66,7\% des migrants disent qu'ils envoyaient l'argent pour couvrir des besoins tels que la scolarité des frères et sœurs, la retraite des parents, le logement et l'alimentation ; 11,11\% des migrants ont déclaré que leur argent permettait à 
leurs proches de se divertir et $11,11 \%$ ont affirmé que l'argent envoyé servait à des fins personnelles.

En ce qui concerne les interactions avec les Français, 31\% des vingt-neuf migrants ont répondu avoir déjà été la cible de propos racistes. La majorité des répondants aux questionnaires échappent donc à la discrimination raciale directe et manifeste contre eux :

J'ai entendu parler du racisme mais je n'ai vraiment pas vécu ça, je ne sais pas si je vais encore le vivre, bien sûr il y a de mes amis qui ont dit que, entre-temps, ils ont eu à saluer des personnes, ils n'ont pas vraiment répondu et tout voilà ce genre de petit truc mais j'ai pas vraiment vécu quelque chose d'aussi grand.

Certaines séquences discriminatoires sont notées par les migrants au quotidien au cours des interactions sociales dans l'espace publique, à l'occasion de la recherche d'un logement, lors des échanges dans la rue, au sein des administrations ou encore dans les rapports de voisinage (Eberhard 482) :

Niveau discrimination, racisme, Dieu merci, j'ai pas été victime de propos raciste direct, mais on sait quand même que le racisme est là mais de manière masquée, de manière un peu déguisée. Par exemple, tu ne verras pas quelqu'un qui va t'attaquer directement en mode " sale noire », mais un exemple, quand tu es dans un bus et que tu vas t'asseoir à côté d'un blanc, y a quelqu'un qui va se lever ; quand tu vas dans un magasin, tu dis bonjour, on ne te répond pas, et quand un blanc rentre et que tu salues on lui répond oui ce genre de petite situations où on sent quand même qu'il y a une discrimination à cause de la couleur de peau ; mais c'est pas direct. (Répondant 4)

Les expressions violentes de racisme, sous forme d'injures ou d'invectives explicites, sont plus rares et racontées comme des situations d'exception sur fond d'idéologie d'extrême droite :

C'est vrai qu'il y a le racisme, c'est plus au nord qu'au sud, je pense, mais comme actuellement ce qu'on voit dans le domaine du foot, oui, on a tout le temps des cris racistes au stade, c'est arrivé déjà en France plusieurs fois déjà et c'est un point qu'on ne doit pas oublier. (Répondant 2)

$\mathrm{Au}$ niveau du contact des cultures, 60\% des migrants pensent que les Français s'intéressent à la culture africaine, maintenue par les jeunes migrants qui intègrent de plus en plus à leur langage les expressions et mots découlant des cultures de leurs parents respectifs. Le contact entre la culture des immigrés africains et la culture française se renforcent également au niveau de la musique et des équipes sportives.

\section{Conclusion}

De nombreux jeunes francophones de l'Afrique de l'Ouest migrent en France par la voie légale dans l'espoir d'améliorer leur éducation et trouver de meilleurs emplois. Cependant, certaines idées préconçues sur la France avant leur départ se sont avérées fausses à leur arrivée : 
les séquences discriminatoires envers les migrants sont rares dans un pays où la vie n'est pas aussi facile qu'ils l'espéraient. Pour la famille ou la collectivité locale, la migration en France continue d'être un investissement eu égard au rapatriement des fonds, alors que la diaspora africaine aide les nouveaux migrants dans leur choix et leur intégration sociale en France. Une fois en France, les migrants avouent avoir rencontré quelques difficultés d'adaptation climatique et sociale, mais être soulagés par la présence de leurs pairs en diaspora.

Si la France reste le lieu de prédilection des migrants francophones de cette étude, il faudrait reconnaitre l'attrait du Canada, des Etats-Unis, du Royaume Uni, de la Chine et même des pays arabes du golfe. Ces migrants, potentiels payeurs d'impôt, représentent un atout que pourraient exploiter les pays qui présentent des conditions d'entrée et des frais de scolarité estudiantine favorables. En considérant le grand pourcentage de francophones en Afrique, la Francophonie tirerait profit du mouvement des jeunes migrants francophones vers la France, plutôt que vers d'autres pays relativement plus attrayants.

\section{Ouvrages cités}

Azizi, SeyedSoroosh. « Altruism: Primary Motivation of Remittances ». Applied Economics Letters 24.17 (2017). 1218-21. www.doi.org/10.1080/13504851.2016.1267840

Barou, Jacques. « Les immigrations africaines en France au tournant du siècle ». Hommes et migrations 1239 (2002). 6-18.

Boyd, Monica. «Family and Personal Networks in International Migration : Recent Developments and New Agendas ». The International Migration Review 23.3 (1989). 638-70.

Boyle, Paul et.al. Exploring Contemporary Migration. Londres : Longman, 1998.

Bréant, Hugo. «Migrations et flux monétaires : quand ceux qui restent financent celui qui part ». Autrepart 4.5 (2013). 31-52.

Diarra, Souleymane. « Les travailleurs africains noirs en France ». BIFAN, tome 30, série B, 3 (1968). 884-1004.

Dumas, Laurent Ribadeau. « Les migrants ouest-africains viennent de pays relativement développés ». France Télévisions, franceinfo : Afrique. 24 juin 2018, mis à jour 23 décembre 2018. https://www.francetvinfo.fr/monde/afrique/societe-africaine/lesmigrants-ouest-africains-viennent-de-pays-relativement-developpes_3054387.html.

Eberhard, Mireille. « De l'expérience du racisme à sa reconnaissance comme discrimination. Stratégies discursives et conflits d'interprétation ». Sociologue 1.4 (2010). 479-95. 
Elbagir, Nima. «People for Sale : Full Documentary ». The CNN Freedom Project. 3 mars 2018. « https://edition.cnn.com/videos/world/2018/03/03/people-for-sale.cnn.

Garson, Jean-Pierre et Anaïs Loizillon. «L'Europe et les migrations de 1950 à nos jours : mutations et enjeux ». Conférence The Economic and Social Aspects of Migration, Commission Européenne et OCDE, Bruxelles, 21-22 janvier 2003.

Gubert, Flaure. « Pourquoi migrer? Le regard de la théorie ». Regards croisés sur l'économie 2.8 (2010). 96-105.

« Immigrés, étrangers ». Insee. 8 octobre 2019.

www.insee.fr/fr/statistiques/3303358? sommaire=3633212.

Lee, Everett S. « A Theory of Migration ». Demography 3.1 (1966). 47-57.

Massey, Douglas et Garcia España. « The Social process of International Migration ». Science 237.4816 (1987). 733-738.

Mouhoud, El Mouhoub. «Les transferts d'épargne des migrants vers les pays d'origine : un enjeu économique pour le développement». La revue d'économie financière: Démographie et finance 122 (2016). 223-237.

« Termes clés de la migration ». International Migration Law No34 - Glossary on Migration. Geneva : Organisation Internationale pour les Migrations (OIM) - UM Migration, 2019. www.iom.int/fr/termes-cles-de-la-migration.

Plazaola, Jean-Philippe de et Elisabeth Rignols, eds. "Tableau de l'économie française ». Collection INSEE Référence, 2018. www.insee.fr/fr/statistiques/3633212.

Quacquarelli, Symonds. «QS World University Ranking@2019». QS Topuniversities, https://www.topuniversities.com/university-rankings/world-university-rankings/2019

Glossaire 2.0 sur l'asile et les migrations : un outil pour une meilleure comparabilité. 2e ed. Luxembourg: Union européenne / Réseau Européen des Migrations (REM), janvier 2012.

Schmitz, Jean. « Migrants ouest-africains vers 1'Europe : historicité et espace moraux ». Politique africaine 1.109 (2008). 5-15.

Stark, Obed et David E. Bloom. « The New Economics of Labor Migration ». The American Economic Review 75.2 (1985). 173-78.

Touré, Niandou. «L'approche « réseaux» dans les études migratoires. Un cas d'étude : les trajectoires scolaires des étudiants maliens en France et au Maroc ». e-Migrinter 13 (2015). 1-14.

Vargas-Lundius, Rosemary, et al. « International Migration, Remittances and Rural Development ». International Fund for Agricultural Development, 2008. 
Vause, Sophie et Sorana Tona. « Peut-on parler de féminisation des flux migratoires du Sénégal et de la République Démocratique du Congo ?». Tr. Camille Richou. Institut national d'études démographique - Population 70.1 (2015). 41-67.

ANNEXE

\begin{tabular}{|c|c|c|c|}
\hline & SEC & & \\
\hline & Valeurs & Fréquence & Pourcentage \\
\hline 1 & Sexe & & \\
\hline & Hommes & 20 & 66,7 \\
\hline & Femmes & 10 & 33,3 \\
\hline & Total & 30 & 100 \\
\hline 2 & Tranches d'âge & & \\
\hline & $15-21$ & 5 & 16,7 \\
\hline & $21-25$ & 18 & 60 \\
\hline & $25-31$ & 5 & 16,7 \\
\hline & $31-50$ & 2 & 3,3 \\
\hline & Total & 30 & 100 \\
\hline 3 & Nationalités : Total (Afrique de l'Ouest) & 30 & 100 \\
\hline 4 & Lieu de naissance Total (Afrique de l'Ouest) & 30 & 100 \\
\hline 5 & Année d'arrivée en France & & \\
\hline & 1998 & 1 & 3,3 \\
\hline & 2009 & 1 & 3,3 \\
\hline & 2013 & 1 & 3,3 \\
\hline & 2014 & 1 & 3,3 \\
\hline & 2015 & 5 & 16,7 \\
\hline & 2016 & 2 & 6,6 \\
\hline & 2017 & 11 & 36,7 \\
\hline & 2018 & 8 & 26,7 \\
\hline & Total & 30 & 100 \\
\hline 6 & Lieu de résidence : France & 30 & 100 \\
\hline 7 & Vous êtes en France pour...? & & \\
\hline & Vos études & & 86,7 \\
\hline & Travailler & & \\
\hline & Retrouver un conjoint & & \\
\hline & Vivre & & \\
\hline & Total & 30 & 100 \\
\hline
\end{tabular}




\begin{tabular}{|c|c|c|c|}
\hline & \multicolumn{3}{|l|}{ SECTION 2} \\
\hline 8 & $\begin{array}{l}\text { Pourquoi avez-vous choisi la France comme pays de } \\
\text { destination? } 30 \text { réponses libres portant sur les similitudes } \\
\text { linguistiques, la langue ainsi que cadre de vie et d'études, la } \\
\text { formation de pointe, le désir de vivre en France, les meilleures } \\
\text { opportunités, trouver du travail, etc. }\end{array}$ & & \\
\hline 9 & $\begin{array}{l}\text { Financement : vous êtes en France ... } \\
\text { A vos propres frais } \\
\text { Aux frais de vos parents/familles/proches } \\
\text { Grace à une bourse } \\
\text { Total }\end{array}$ & $\begin{array}{l}8 \\
19 \\
3 \\
30\end{array}$ & $\begin{array}{l}26,7 \\
63,3 \\
10 \\
100\end{array}$ \\
\hline 10 & Moyens de transport : Comment êtes-vous arrivés en France ? & & \\
\hline & $\begin{array}{l}\text { Par voie aérienne (avion) } \\
\text { Par la mer } \\
\text { Par la route } \\
\text { Vous ne voulez pas en parler } \\
\text { Total }\end{array}$ & $\begin{array}{l}28 \\
0 \\
1 \\
1 \\
30\end{array}$ & $\begin{array}{l}93,3 \\
0 \\
3,3 \\
3,3 \\
100,0\end{array}$ \\
\hline 11 & $\begin{array}{l}\text { Aviez-vous pris la décision vous-même de partir? } \\
\text { Oui } \\
\text { Non } \\
\text { Total }\end{array}$ & $\begin{array}{l}23 \\
6 \\
29\end{array}$ & $\begin{array}{l}80 \\
20 \\
100,0\end{array}$ \\
\hline 12 & $\begin{array}{l}\text { Vos parents ont-ils influencé cotre décision? } \\
\text { Oui } \\
\text { Non } \\
\text { Partiellement } \\
\text { Total }\end{array}$ & $\begin{array}{l}8 \\
15 \\
7 \\
30\end{array}$ & $\begin{array}{l}26,7 \\
50 \\
23,3 \\
100\end{array}$ \\
\hline 13 & $\begin{array}{l}\text { Lesquels de ces raisons vous ont poussé à quitter votre pays } \\
\text { d'origine ? (N.B. : réponses multiples par les } 30 \text { répondants) } \\
\text { L'instabilité politique } \\
\text { La pauvreté } \\
\text { Le désir d'effectuer une formation de pointe } \\
\text { Le goût de l'aventure } \\
\text { Avoir accès à de meilleurs soins de santé }\end{array}$ & $\begin{array}{l}6 \\
4 \\
22 \\
4 \\
4\end{array}$ & $\begin{array}{l}20 \\
13,3 \\
73,3 \\
13,3 \\
13,3\end{array}$ \\
\hline 14 & $\begin{array}{l}\text { Etes-vous parti pour obtenir des choses auxquelles vous } \\
\text { n'aviez pas accès dans votre pays d'origine? }\end{array}$ & & \\
\hline
\end{tabular}




\begin{tabular}{|c|c|c|c|}
\hline & $\begin{array}{l}\text { Oui } \\
\text { Non } \\
\text { Peut-être } \\
\text { Total }\end{array}$ & $\begin{array}{l}20 \\
3 \\
7 \\
30\end{array}$ & $\begin{array}{l}66,7 \\
10 \\
23,33 \\
100\end{array}$ \\
\hline 15 & $\begin{array}{l}\text { Pensez-vous que de meilleures opportunités s'offrent à vous en } \\
\text { France? } \\
\text { Oui } \\
\text { Non } \\
\text { Total }\end{array}$ & $\begin{array}{l}27 \\
3 \\
30\end{array}$ & $\begin{array}{l}90 \\
10 \\
100\end{array}$ \\
\hline 16 & $\begin{array}{l}\text { Si Oui, pourquoi ? (21 réponses libres) : la France est belle, } \\
\text { stable, offre d'emplois, formation spéciale, infrastructures, } \\
\text { égalité, bonne rémunération }\end{array}$ & 21 & 100 \\
\hline 17 & $\begin{array}{l}\text { Admettons que vous avez dans votre pays d'origine les mêmes } \\
\text { opportunités que celles qui vous sont offertes en France, } \\
\text { seriez-vous restés en Afrique? } \\
\text { Oui } \\
\text { Non } \\
\text { Peut-être } \\
\text { total }\end{array}$ & $\begin{array}{l}21 \\
4 \\
5 \\
30\end{array}$ & $\begin{array}{l}70 \\
13,3 \\
16,7 \\
100\end{array}$ \\
\hline 18 & $\begin{array}{l}\text { Comptez-vous vous installer définitivement en France? } \\
\text { Oui } \\
\text { Non } \\
\text { Total }\end{array}$ & $\begin{array}{l}24 \\
6 \\
30\end{array}$ & $\begin{array}{l}80 \\
20 \\
100\end{array}$ \\
\hline 19 & $\begin{array}{l}\text { Vous êtes-vous représenté la France d'une quelconque } \\
\text { manière avant d'y arriver? } \\
\text { Oui } \\
\text { Non } \\
\text { Total }\end{array}$ & $\begin{array}{l}7 \\
23 \\
30\end{array}$ & $\begin{array}{l}23,3 \\
76,7 \\
100\end{array}$ \\
\hline 20 & $\begin{array}{l}\text { Si Oui, quelles sont ces représentations et se sont-elles avérées } \\
\text { vraies? } 5 \text { réponses libres : }\end{array}$ & 3 & 60 \\
\hline
\end{tabular}




\begin{tabular}{|c|c|c|c|}
\hline & $\begin{array}{l}\text { Vrai : niveau normal de recherche ; climat difficile ; beau pays, } \\
\text { bons profs, diversité culturelle, facilité de vie, individualisme } \\
\text { Partiellement vrai : racisme } \\
\text { Faux : un pays facile } \\
\text { Total }\end{array}$ & $\begin{array}{l}1 \\
1 \\
5\end{array}$ & $\begin{array}{l}20 \\
20 \\
100\end{array}$ \\
\hline 21 & $\begin{array}{l}\text { Rester en France, travailler pour pouvoir vous occuper de vos } \\
\text { parents plus tard fait-il parti de vos objectifs? } \\
\text { Oui } \\
\text { Non } \\
\text { Total }\end{array}$ & 30 & $\begin{array}{l}53,3 \\
46,7 \\
100\end{array}$ \\
\hline 22 & $\begin{array}{l}\text { Vos proches (amis, famille, parents) se trouvaient-ils en } \\
\text { France avant votre arrivée? } \\
\text { Oui } \\
\text { Non } \\
\text { Total }\end{array}$ & $\begin{array}{l}20 \\
9 \\
29\end{array}$ & $\begin{array}{l}69 \\
31 \\
100\end{array}$ \\
\hline 23 & $\begin{array}{l}\text { Si oui, le fait que vos proches se trouvent en France aurait-il } \\
\text { influencé le choix de votre destination? } \\
\text { Oui } \\
\text { Non } \\
\text { Total }\end{array}$ & 21 & $\begin{array}{l}57,1 \\
42,9 \\
100\end{array}$ \\
\hline 24 & $\begin{array}{l}\text { Vous ont-ils aidé d'une manière quelconque à votre arrivée? } \\
\text { Oui } \\
\text { Non } \\
\text { Total }\end{array}$ & $\begin{array}{l}14 \\
8 \\
26\end{array}$ & $\begin{array}{l}69,2 \\
30,8 \\
100\end{array}$ \\
\hline 25 & $\begin{array}{l}\text { Si Oui, comment ? } 14 \text { réponses libres portant sur l'hébergement, } \\
\text { l'intégration, les démarches administratives, les renseignements, } \\
\text { frais de voyage, etc. }\end{array}$ & & \\
\hline 26 & $\begin{array}{l}\text { Savoir qu'ils sont présents vous a-t-il réconforté d'une } \\
\text { quelconque manière? } \\
\text { Oui } \\
\text { Non } \\
\text { Total }\end{array}$ & $\begin{array}{l}20 \\
5 \\
25\end{array}$ & $\begin{array}{l}80 \\
20 \\
100\end{array}$ \\
\hline
\end{tabular}




\begin{tabular}{|c|c|c|c|}
\hline 27 & $\begin{array}{l}\text { Travaillez-vous ? (Emploi à plein temps, mi-temps, saisonnier, } \\
\text { jobs, business, etc.) } \\
\text { Oui } \\
\text { Non } \\
\text { Total }\end{array}$ & $\begin{array}{l}14 \\
16 \\
30\end{array}$ & $\begin{array}{l}46,7 \\
53,3 \\
100\end{array}$ \\
\hline 28 & $\begin{array}{l}\text { Si Oui, auriez-vous déjà envoyé de l'argent à un membre de } \\
\text { votre famille? } \\
\text { Oui } \\
\text { Non } \\
\text { Total }\end{array}$ & $\begin{array}{l}11 \\
13 \\
24\end{array}$ & $\begin{array}{l}45,8 \\
54,2 \\
100\end{array}$ \\
\hline 29 & $\begin{array}{l}\text { A quelle fréquence envoyez-vous de l'argent? } \\
\text { Mensuellement } \\
\text { Occasionnellement } \\
\text { Rarement } \\
\text { Jamais } \\
\text { Total }\end{array}$ & $\begin{array}{l}4 \\
4 \\
1 \\
1 \\
10\end{array}$ & $\begin{array}{l}40 \\
40 \\
10 \\
10 \\
100\end{array}$ \\
\hline 30 & $\begin{array}{l}\text { Quelles sont les raisons vous poussant à leur envoyer de } \\
\text { l'argent ? } 10 \text { réponses libres portant sur } \\
\text { Aide financière à la famille } \\
\text { Dons } \\
\text { Personnelles } \\
\text { Aucune } \\
\text { Total }\end{array}$ & $\begin{array}{l}6 \\
2 \\
1 \\
1 \\
10\end{array}$ & $\begin{array}{l}60 \\
20 \\
10 \\
10 \\
100\end{array}$ \\
\hline 31 & $\begin{array}{l}\text { À quoi sert l'argent que vous envoyez ? } 9 \text { réponses libres sur } \\
\text { Besoins de première nécessité pour la famille } \\
\text { Divertissement } \\
\text { Fins personnels } \\
\text { Aucune idée } \\
\text { Total }\end{array}$ & $\begin{array}{l}6 \\
1 \\
1 \\
1 \\
9\end{array}$ & $\begin{array}{l}66,7 \\
11,1 \\
11,1 \\
11,1 \\
100\end{array}$ \\
\hline 32 & $\begin{array}{l}\text { Quels obstacles avez-vous rencontrés à votre arrivée en } \\
\text { France ? } 21 \text { réponses sur le froid, aliments, parents, dépaysement, } \\
\text { dépression, isolement, nouvelle mentalité, logement, } \\
\text { administratif, etc. }\end{array}$ & & \\
\hline
\end{tabular}




\begin{tabular}{|c|c|c|c|}
\hline & $\begin{array}{l}\text { Oui } \\
\text { Non } \\
\text { Total }\end{array}$ & $\begin{array}{l}9 \\
20 \\
29\end{array}$ & $\begin{array}{l}31 \\
69 \\
100\end{array}$ \\
\hline 34 & $\begin{array}{l}\text { Quels sont vos rapports avec les Français? } \\
\text { Assez bien } \\
\text { Bien } \\
\text { Vous ne voulez pas en parler } \\
\text { Total }\end{array}$ & $\begin{array}{l}17 \\
12 \\
1 \\
30\end{array}$ & $\begin{array}{l}56,7 \\
40 \\
3,3 \\
100\end{array}$ \\
\hline 35 & $\begin{array}{l}\text { Montrent-ils de l'intérêt envers la culture Africaine? } \\
\text { Oui } \\
\text { Non } \\
\text { Total }\end{array}$ & $\begin{array}{l}18 \\
12 \\
30\end{array}$ & $\begin{array}{l}60 \\
40 \\
100\end{array}$ \\
\hline 36 & $\begin{array}{l}\text { Finalement, comptez-vous de repartir dans votre pays } \\
\text { d'origine et vous y installer un de ces jours? } \\
\text { Non } \\
\text { Oui } \\
\text { Total }\end{array}$ & $\begin{array}{l}7 \\
23 \\
30\end{array}$ & $\begin{array}{l}23,3 \\
76,7 \\
100\end{array}$ \\
\hline 37 & $\begin{array}{l}\text { Si Oui, pourquoi ? } 21 \text { réponses portant sur l'amour de la patrie et } \\
\text { la contribution au développement de leur pays d'origine }\end{array}$ & & \\
\hline
\end{tabular}

